\title{
Sildenafil no tratamento da hipertensão pulmonar associada a lúpus eritematoso sistêmico e síndrome antifosfolipídio*
}

\author{
Sildenafil for treatment of pulmonary hypertension in association \\ with systemic lupus erythematosus and anti-phospholipid syndrome
}

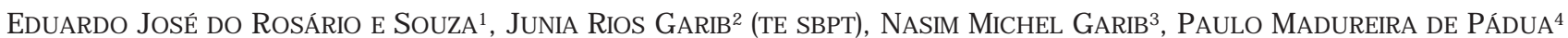

Hipertensão pulmonar grave é uma doença debilitante, com expectativa de vida reduzida, que acomete adultos jovens. Complicações pleuropulmonares no lúpus eritematoso sistêmico ocorrem em $50 \%$ a $70 \%$ dos pacientes. A hipertensão pulmonar grave no lúpus eritematoso sistêmico é rara e tem prognóstico reservado. Descreve-se, pela primeira vez, um paciente com hipertensão pulmonar grave associada a lúpus eritematoso sistêmico e síndrome antifosfolipídio secundário que apresentou boa resposta ao uso do sildenafil oral, após falha do tratamento convencional com corticosteróides, ciclofosfamida, warfarin e diltiazem. (J Pneumol 2003;29(5):302-4)

Descritores - Vasodilatadores/ uso terapêutico. Lúpus eritematoso sistêmico. A nticorpos antifosfolipídios. Inibidores da fosfodiesterase.
Severe pulmonary hypertension is a debilitating disease with short life expectancy that often affects young people. Pleuropulmonary complications of systemic lupus erythematosus occur in $50-70 \%$ of patients. Severe symptomatic pulmonary hypertension in systemic lupus erythematosus is rare and carries a bad prognosis because a fatal outcome can occur within months. The authors describe, for the first time, a patient with systemic lupus erythematosus with severe pulmonary hypertension and secondary antiphospholipid syndrome who responded favorably to oral sildenafil, after unsuccessful use of prednisone, intravenous cyclophosphamide, warfarin and diltiazem.

Key words - Vasodilator agents/ therapeutic use. Lupus erythematosuos systemic. Antiphospholipid antibodies. Phosphodiesterase inhibitors.

\section{Veja cometários no Editorial, na página 251}

\section{INTRODUÇÃO}

0 termo hipertensão pulmonar ( $\mathrm{H} P$ ) refere-se a um grupo de doenças caracterizadas por elevação da pressão da artéria pulmonar, a qual tem como determinantes a resistência vascular pulmonar, o débito cardíaco e a pressão do átrio esquerdo. A doença pode ser primária ou secundária. (1) Doenças difusas do tecido conjuntivo podem ocasionalmente evoluir com a presença de HP. Assim como a
Siglas e abreviaturas utilizadas neste trabalho

GMPC - Monofosfato cíclico de guanosina

HP - Hipertensão pulmonar

HPP - Hipertensão pulmonar primária

LES - Lúpus eritematoso sistêmico

NO - Óxido nítrico

PDE5 - Fosfodiesterase 5

SAAF - Síndrome do anticorpo antifosfolipídio

TCAR - Tomografia computadorizada de alta resolução
* Trabalho realizado no Hospital da Santa Casa de Belo Horizonte.

1. Mestre em Clínica Médica. Coordenador da Especialização em Reumatologia do Hospital da Santa Casa de Belo Horizonte.

2. Médica Assistente da IV Clínica Médica do Hospital da Santa Casa de B elo Horizonte. Professora A uxiliar de Pneumologia da Faculdade de Ciências Médicas de Minas Gerais. Título de especialista pela Sociedade Brasileira de Pneumologia e Tisiologia.

3. Coordenador da IV Clínica Médica do Hospital da Santa Casa de Belo Horizonte. Professor Titular de Pneumologia da Faculdade de Ciências Médicas de Minas Gerais.
4. Coordenador do Serviço de Reumatologia do H ospital da Santa Casa de Belo Horizonte. Professor Titular de Reumatologia da Universidade Federal de Minas Gerais.

Endereço para correspondência - Eduardo J osé do Rosário e Souza, Santa Casa de B elo H orizonte, Serviço de Reumatologia da Santa Casa de Belo Horizonte, Av. Francisco Sales, 1.111, 9o andar, ala B - 30150221 - Belo Horizonte, MG - Brasil. E-mail: souzaeduardo@uaimail. com.br

Recebido para publicação em 20/3/03. Aprovado, após revisão, em 15/6/03. 
hipertensão pulmonar primária, a hipertensão pulmonar secundária também pode afetar significativamente a qualidade de vida e aproximar a morte em pacientes com doenças do tecido conjuntivo.(2)

A associação de hipertensão pulmonar grave com a presença de anticorpos antifosfolipídios foi descrita pela primeira vez em 1983, quando 5 de 6 pacientes portadores de lúpus eritematoso e hipertensão pulmonar apresentaram anticoagulante lúpico positivo. A hipertensão pulmonar grave sintomática no lúpus eritematoso sistêmico (LES) é rara e, quando presente, acarreta um prognóstico ruim, com resultados fatais que podem ocorrer em poucos meses, geralmente por colapso circulatório. (3)

As opções de tratamento são limitadas na HP grave. Relatos recentes e alguns estudos preliminares têm indicado que o sildenafil, um inibidor específico da 5 fosfodiesterase, largamente usado no tratamento da disfunção erétil, reduz a resistência vascular pulmonar em humanos com HPP, em voluntários sadios com vasoconstrição pulmonar induzida por hipoxemia e em animais com hipertensão pulmonar grave experimental.(4)

Descrevemos abaixo uma paciente portadora de hipertensão pulmonar associada a LES associado a síndrome antifosfolipídio (SAAF) refratária aos tratamentos convencionais e que apresentou resposta favorável após o uso de sildenafil.

\section{RELATO DO CASO}

Mulher de 33 anos de idade, acompanhada pelos Serviços de Reumatologia e Pneumologia da Santa Casa de B elo H orizonte, ap resentou eritema malar, derrame pleural, artrite em mãos e joelhos, proteinúria e fator antinuclear positivo durante a gravidez, recebendo diagnóstico de LES. Não havia história de tabagismo ou etilismo. $\mathrm{Na}$ época do diagnóstico relatava dor opressiva em região torácica anterior e dispnéia de grau II. No exame clínico, apresentava a segunda bulha cardíaca desdobrada e hiperfonética, e pressão arterial sistêmica normal. Os exames complementares demonstraram: pesquisa de anticoagulante lúpico positiva, radiografia de tórax com proeminência do arco da artéria pulmonar, espirometria com distúrbio ventilatório restritivo leve, TCAR normal, eletrocardiograma com sobrecarga de câmaras direitas, ecodopplercardiograma transtorácico com dilatação de câmaras cardíacas direitas associada a disfunção de ventrículo direito e valor de pressão sistólica de artéria pulmonar (PAP) de $74 \mathrm{mmH}$ g, cintilografia pulmonar com a relação ventilação/ perfusão normal, gasometria arterial normal, duplex scan venoso de membros inferiores com presença de trombos proximais (assintomática) e tomografia computadorizada helicoidal normal. Com base nesses achados, foi feito o diagnóstico de HP associada a LES e SAAF.

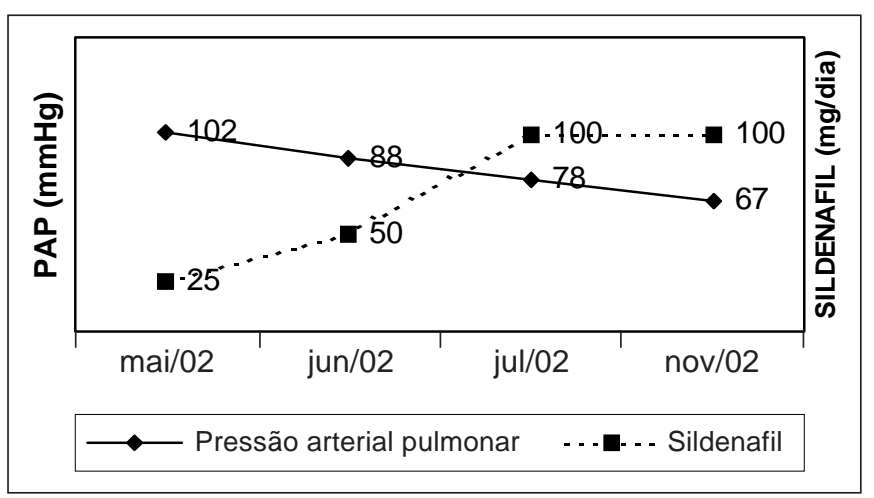

Figura 1 - Declínio dos níveis de pressão arterial pulmonar sistólica após o início do sildenafil

A paciente foi submetida ao seguinte esquema de tratamento: prednisona $1 \mathrm{mg} / \mathrm{kg} /$ dia (com posterior redução gradual), difosfato de cloroquina $4 \mathrm{mg} / \mathrm{kg} / \mathrm{dia}$, diltiazem $240 \mathrm{mg} / \mathrm{dia}$, warfarin oral para anticoagulação e ciclofosfamida sob a forma de pulsos mensais. 0 uso do diltiazem foi empírico, pois a paciente se recusou a realizar o teste de vasodilatação com monitoração invasiva da pressão arterial pulmonar. A pós seis meses de tratamento, o ecodopplercardiograma revelava pressão sistólica estimada em artéria pulmonar de $80 \mathrm{mmHg}$. 0 uso de derivados da prostaciclina (iloprost) não foi possível devido a seu alto custo. Durante a evolução, foram realizados dois novos ciclos com seis meses de duração de pulsoterapia com metilprednisolona e ciclofosfamida com seis meses de duração, porém, a pressão sistólica arterial pulmonar, aferida pelo ecodop plercardiograma, não respondeu, chegando a atingir seu maior valor, de $102 \mathrm{mmH} \mathrm{g}$. Caracterizada a falta de resposta aos tratamentos convencionais, optamos pelo uso de sildenafil oral.

Com base em indicativos favoráveis na literatura médica, após a obtenção da aprovação da Comissão de Ética do Hospital da Santa Casa de Belo Horizonte e da assinatura do termo de consentimento livre e esclarecido pela paciente, iniciamos o uso do sildenafil via oral na dose de $25 \mathrm{mg}$ ao dia, com elevação gradual até $100 \mathrm{mg}$ ao dia em duas tomadas. A pressão sistólica na artéria pulmonar foi estimada pelo ecodopplercardiograma, havendo queda progressiva e sustentada durante os seis meses de uso do medicamento, chegando a 34\% de declínio (Figura 1). Não houve relato de efeitos colaterais. A paciente permaneceu em uso de prednisona $5 \mathrm{mg}$, difosfato de cloroquina $160 \mathrm{mg}$ e warfarin $5 \mathrm{mg}$ ao dia.

\section{DISCUSSÃO}

A HP grave é uma doença debilitante que resulta em curta expectativa de vida e que freqüentemente afeta indivíduos jovens. ${ }^{(5)}$ É de ocorrência rara a presença de hi- 
pertensão pulmonar grave no LES, porém casos leves subclínicos não são incomuns. Embora a patogênese da HP grave no LES não seja bem compreendida, múltiplos fatores etiológicos estão provavelmente envolvidos, dentre os quais o fenômeno tromboembólico recorrente crônico, a trombose pulmonar in situ devida à presença de anticorpos antifosfolipídios, e uma possível disfunção endotelial com resposta vascular anormal. A evolução dos pacientes com HP grave depende da intensidade da doença. Taxas de mortalidade avaliadas em um período acima de 5 anos variam entre $14 \%$ e $50 \%$, sendo usual a morte súbita em decorrência de colapso circulatório. 0 tratamento medicamentoso é realizado empiricamente e as respostas têm sido insatisfatórias. 0 uso de corticosteróides e imunossupressores não costuma ser efetivo em pacientes com HP primária, porém pacientes com hipertensão pulmonar secundária ao LES têm mostrado resposta melhor ao uso desses agentes terapêuticos. (3)

A limitação em relação ao uso de agentes vasodilatadores na hipertensão pulmonar é atribuída, em parte, à falta de potência e de seletividade desses medicamentos em relação à circulação pulmonar. Entretanto, avanços significativos têm ocorrido nos últimos anos. O sildenafil, um potente e seletivo inibidor da PDE5, é mais bem conhecido pelo seu uso no tratamento da disfunção erétil. A lém de sua elevada concentração nos corpos cavernoSOS, a PDE5 é abundante em vasos, na traquéia, na musculatura lisa visceral e em plaquetas. 0 tratamento de longo prazo (três meses) com sildenafil oral melhorou a capacidade de realizar exercícios e a qualidade de vida em pacientes com hipertensão pulmonar grave. ${ }^{(6)}$ Michekalis et al. ${ }^{(4)}$ demonstraram que o sildenafil oral em dose única é um potente e seletivo vasodilatador pulmonar. 0 sildenafil foi também utilizado com resultados satisfatórios em dois casos descritos por Watanabe et al.,(2) uma paciente portadora de HP primária e a segunda, de HP associada a doença difusa do tecido conjuntivo.

Descrevemos, pela primeira vez, um caso de HP grave associada a LES e SAAF, que apresentou boa resposta ao sildenafil oral, de acordo com indicativos favoráveis da literatura médica. A creditamos que o uso do sildenafil pode ser uma opção de tratamento em pacientes portadores de hipertensão pulmonar secundária a doenças do tecido conjuntivo que não respondam ao método convencional. Mais trabalhos para avaliar a segurança e a eficácia do sildenafil oral em pacientes com hipertensão pulmonar primária e secundária serão necessários para assegurar 0 seu uso nesta condição clínica.

\section{REFERÊNCIAS}

1. Dweik RA. Pulmonary hypertension and the search for the selective pulmonary vasodilator. Lancet 2002;360:886-7.

2. Watanabe H, Ohashi K, Takeuchi K, Yamashita K, Yokoyama T, Tran $\mathrm{OK}$, et al. Sildenafil for primary and secondary pulmonary hypertension. Clin Pharmacol Ther 2002;71:398-402.

3. Tam L-S, Li EK. Successful treatment with immunosuppression, anticoagulation and vasodilator therapy of pulmonary hypertension in SLE associated with secondary antiphospholipid syndrome. Lupus 1998;7: 495-7.

4. Michelakis E, Tymchak W, Lien D, Webster L, Hashimoto K, Archer S. Oral sildenafil is an effective and specific pulmonary vasodilator in patients with pulmonary arterial hypertension. Circulation 2002;105:2398403.

5. Ghofrani HA, Wieddemann R, Rose F, Alschewski H, Schermuly RT, Weissmann $\mathrm{N}$, et al. Combination therapy with oral sildenafil and inhaled iloprost for severe pulmonary hypertension. Ann Intern Med 2002; 136:515-22

6. Lodato RF. Viagra for impotence of pulmonary vasodilator therapy? Am J Respir Crit Care Med 2001;163:312-3. 